\title{
The links between hypertrophy, reproductive potential and longevity in the Saccharomyces cerevisiae yeast*
}

\author{
Mateusz Molon ${ }^{凶}$ and Renata Zadrag-Tecza \\ Department of Biochemistry and Cell Biology, University of Rzeszow, Rzeszów, Poland
}

\begin{abstract}
The yeast Saccharomyces cerevisiae has long been used as a model organism for studying the basic mechanisms of aging. However, the main problem with the use of this unicellular fungus is the unit of "longevity". For all organisms, lifespan is expressed in units of time, while in the case of yeast it is defined by the number of daughter cells produced. Additionally, in yeast the phenotypic effects of mutations often show a clear dependence on the genetic background, suggesting the need for an analysis of strains representing different genetic backgrounds. Our results confirm the data presented in earlier papers that the reproductive potential is strongly associated with an increase in cell volume per generation. An excessive cell volume results in the loss of reproductive capacity. These data clearly support the hypertrophy hypothesis. The time of life of all analysed mutants, with the exception of sch9 $\Delta$, is the same as in the case of the wild-type strain. Interestingly, the $121 \%$ increase of the fob1 $\Delta$ mutant's reproductive potential compared to the sfp $1 \Delta$ mutant does not result in prolongation of the mutant's time of life (total lifespan).
\end{abstract}

Key words: yeast, lifespan, longevity, hypertrophy

Received: 01 August, 2015; revised: 17 January, 2016; accepted: 11 February, 2016; available on-line: 29 February, 2016

\section{INTRODUCTION}

Aging is defined as "any age-specific decline in variables associated with individual fitness, specifically mortality, reproduction and physiological performance" (Reznick et al., 2004). Despite the complicated etiology of the process, we still lack certainty as to which factors lead to longevity. The choice of an appropriate model for research has been a challenge for numerous scientists. Model organisms used in many fields of science, such as medicine, biology or biotechnology, should help overcomes experimental constraints associated with ethical use of a host organism, including the human being. They are also useful in developing and optimising analytical methods. The important thing is that such organisms should be representative of a larger class of living forms. Unfortunately, the use of a specific model organism does not always ensure that the same results will be obtained in other organisms (Karathia et al., 2011). From among the variety of types of such organisms, the $S$. cerevisiae yeast was proposed as a model organism for explanation of the general mechanisms of aging (Bitterman et al., 2003; Jazwinski, 1999; Piper, 2006). The use of yeast in the studies of aging has its clear advantages, such as the small genome, short cell cycle, numerous protocols and broad knowledge of this unicellular eukaryotic species. Yeast is described in detail also at the molecular level. We can use successfully various tools to change and monitor cellular processes, such as translation and transcription. On the other hand, we should also take into consideration certain problems or limitations resulting from the use of this particular unicellular organism. An example of such consideration is homeostatic regulation of both intra- and extracellular environments by yeast and higher (multicellular) eukaryotes. Yeast regulates its intracellular processes in a way similar to other eukaryotes. However, as a unicellular organism yeast is more susceptible to environmental factors (changes of temperature, nutrients, metabolites and other) which can regulate its metabolism, as opposed to multicellular organisms where cells have a relatively constant external environment. Other controversies include absence of DNA silencing by methylation, lack of miRNA processing machinery or lack of telomere shortening during successive replication cycles (Gershon \& Gershon, 2000). Furthermore, the amount of non-coding DNA and only marginal contribution of alternative splicing indicate that there are aspects where yeast biology is incomparable to the biology of higher eukaryotes. Apart from the differences associated with molecular, physiological or phylogenetic relations between yeast and higher eukaryotic organisms, there are important limitations concerning the way of expressing their lifespan. In the case of yeast, this parameter is defined as the number of daughter cells produced (Sinclair et al., 1998), whereas for other organisms lifespan is expressed in units of time. Our recent studies with the use of the so called "longevity mutants" (organisms with increased reproductive potential) showed no significant differences in their time of life (total lifespan). In spite of significant differences in the reproductive potential, the total lifespan of the mutants remained almost identical to that of the wild-type strain (Molon et al., 2015).

The phenomenon discovered by Mortimer and Johnston (Mortimer \& Johnston, 1959) was termed replicative lifespan (RLS) and defined as the number of daughter cells produced by a mother cell (Kaeberlein, 2010), later named reproductive potential (Zadrag et al., 2008). Mortimer and Johnston were the first to show that yeast cells can perform a limited number of cycles (Mortimer \& Johnston, 1959). During that time, a lot of theories emerged describing various factors that might affect the numeric value of the replicative lifespan. One of the first candidates proposed for the role of the "senescence factor" was extrachromosomal rDNA circles (Sinclair

e-mail: mateuszmolon@univ.rzeszow.pl

*The results were presented at the 6th International Weigl Conference on Microbiology, Gdańsk, Poland (8-10 July, 2015).

Abbreviations: ERCs, extrachromosomal rDNA circles; RLS, replicative lifespan 
\& Guarente, 1997). The role of the "senescence factor" was also attributed to oxidatively damaged proteins (Aguilaniu et al., 2003) or thermal aggregates (Erjavec et al., 2007). Replicative lifespan may also be influenced by various genes and environmental conditions (Steinkraus et al., 2008). For many years, the flagship "longevity mutant" was fob1 $\triangle$. FOB1 (fork blocking less) is a gene that has no homologues in other organisms. The Fob1 protein occurs in the nucleolus (Defossez et al., 1999). Blocking the replication forks by mutations in the FOB1 gene proved to be an important factor in reducing the number of extrachromosomal rDNA circles (ERCs), which were regarded as one of the many "senescence factors". Studies have shown that ERCs accumulate in larger amounts in old cells; for cells this means that they have performed a lot of reproductive cycles. The increased number of ERCs was postulated to decrease the reproductive potential of yeast cells (Sinclair \& Guarente, 1997). Another gene, the mutation of which leads to an increased reproductive potential, is $S \mathrm{CH}$ 9. The yeast SCH9 gene encodes a serine/threonine kinase involved in the glucose response and regulation of transcription by RNA polymerases I, II, and III. Sch9p also performs a function analogous to the mammalian S6K gene (Urban et al., 2007). Mutation in this gene extends chronological lifespan and causes an increase in the reproductive potential. It also causes an increased resistance to oxidative and thermal stress (Fabrizio et al., 2001; Kaeberlein \& Kennedy, 2005). With yeast as a model organism used for studies on the aging process, much research has been devoted to the analysis of replicative lifespan, which refers only to the number of daughter cells produced. On that basis, numerous of "longevity mutants" were proposed. However, when in addition to the number of daughters we also consider the time of life, the only longevity yeast mutant (for longevity expressed in units of time) would be the one that has been recently described as sfp1A (Molon et al., 2015). The product of the SFP1 gene is a protein (split finger-zinc protein) that plays a key role in regulating the biosynthesis of ribosomal proteins. This protein can also have an impact on reproductive potential (Heeren et al., 2009; Molon et al., 2015).

An alternative postulate is that the limit of mitotic cycles is not a consequence of accumulation of a "senescence factor" but results from cell achieving its critical volume, which prevents further reproduction (Yang et al., 2011; Zadrag-Tecza et al., 2009). Studies concerning the impact of genes on RLS often face the problem of the impact of the genetic background on the phenotypic effect of deletion of selected genes, which makes it difficult to formulate clear conclusions. Therefore, it is important in this case to take into account other parameters, such as changes in cell size or duration of various phases in yeast cell life (reproductive and post-reproductive) because such parameters may facilitate interpretation of the differences observed in the studied yeast strains related to genetic backgrounds.
The aim of this work is to analyse the reproductive potential and time of life (expressed in time units) of yeast strains devoid of the genes known as "longevity genes", namely FOB1, SCH9 and SFP1, and also to verify whether there is a relationship between the excessive volume of cells (hypertrophy) and reproductive potential. The role of cell size in the regulation of reproductive potential of yeast was presented in our team's previous papers. As the topic has recently spurred heated discussion, it is worth adding some further data. In addition, the new aspect of these studies was using the BMA64-1A genetic background with cells achieving larges sizes in both mean and maximum terms, in comparison with cells representing other genetic backgrounds.

\section{MATERIALS AND METHODS}

Chemicals. Components of culture media were from BD Difco (Becton Dickinson and Company, Spark), except for glucose (POCh, Gliwice, Poland).

Yeast strains. Strains used for the purpose of this paper are show in Table 1. The strains were constructed as described in Molon et al., 2015.

Media and growth conditions. Yeast was grown in a standard liquid YPD medium (1\% yeast extract, 1\% yeast Bacto-peptone, $2 \%$ glucose) on a rotary shaker at $150 \mathrm{rpm}$ at a temperature of $28^{\circ} \mathrm{C}$.

Determination of reproductive potential. Reproductive potential of yeast (the number of daughter produced by a single cell during its life) was determined according to the previously described procedure (Wawryn et al., 1999). Five microliter aliquots of culture from the exponential phase of growth $\left(5 \times 10^{-7}\right.$ cells $/ \mathrm{ml}$ density) was dropped on separate YPD plates. Forty single cells, randomly chosen, were used for each experiment. The analysis was performed by micromanipulation using the Nikon Eclipse E200 optical microscope with the attached micromanipulator. The number of buds formed by each cell signifies the reproductive potential of the cell. During the manipulation, the plates were kept at $28^{\circ} \mathrm{C}$ for $16 \mathrm{~h}$ and at $4^{\circ} \mathrm{C}$ during the night. The data represent the mean values from three separate experiments.

Determination of reproductive lifespan, post-reproductive lifespan and total lifespan. Yeast lifespan was determined according to the previously described method (Minois et al., 2005) with some modifications (Zadrag et al., 2008). Five microliter aliquots of culture from the exponential phase of growth $\left(5 \times 10^{-7}\right.$ cells/ $\mathrm{ml}$ density) was dropped on separate YPD plates with solid medium containing Phloxine B $(10 \mu \mathrm{g} / \mathrm{ml})$. Forty single cells were used for each experiment. The analysis was performed by micromanipulation using the Nikon Eclipse E200 optical microscope with the attached micromanipulator. Phloxine B was used as the indicator of dead yeast cells during determination of the total lifespan of cells. The total lifespan was calculated as the sum of the reproductive and post-reproductive lifespans (each

Table 1. Strains used in this study

\begin{tabular}{lll}
\hline Strain & Genotype & Source \\
\hline BMA64-1A (wild-type) & MAT a ura3-1 trp1 $\Delta 2$ leu2-3,112 his3-11,15 ade2-1 can1-100 & Euroscarf \\
\hdashline fob1 $\Delta$ & MAT a ura3-1 trp1 $\Delta 2$ leu2-3,112 his3-11,15 ade2-1 can1-100 fob1::kanMX4 & in this study \\
\hline sch9 $\Delta$ & MAT a ura3-1 trp1 $\Delta 2$ leu2-3,112 his3-11,15 ade2-1 can1-100 sch9::kanMX4 & in this study \\
\hdashline$s p 1 \Delta$ & MAT a ura3-1 trp1 $\Delta 2$ leu2-3,112 his3-11,15 ade2-1 can1-100 sfp1::kanMX4 & in this study \\
\hline
\end{tabular}



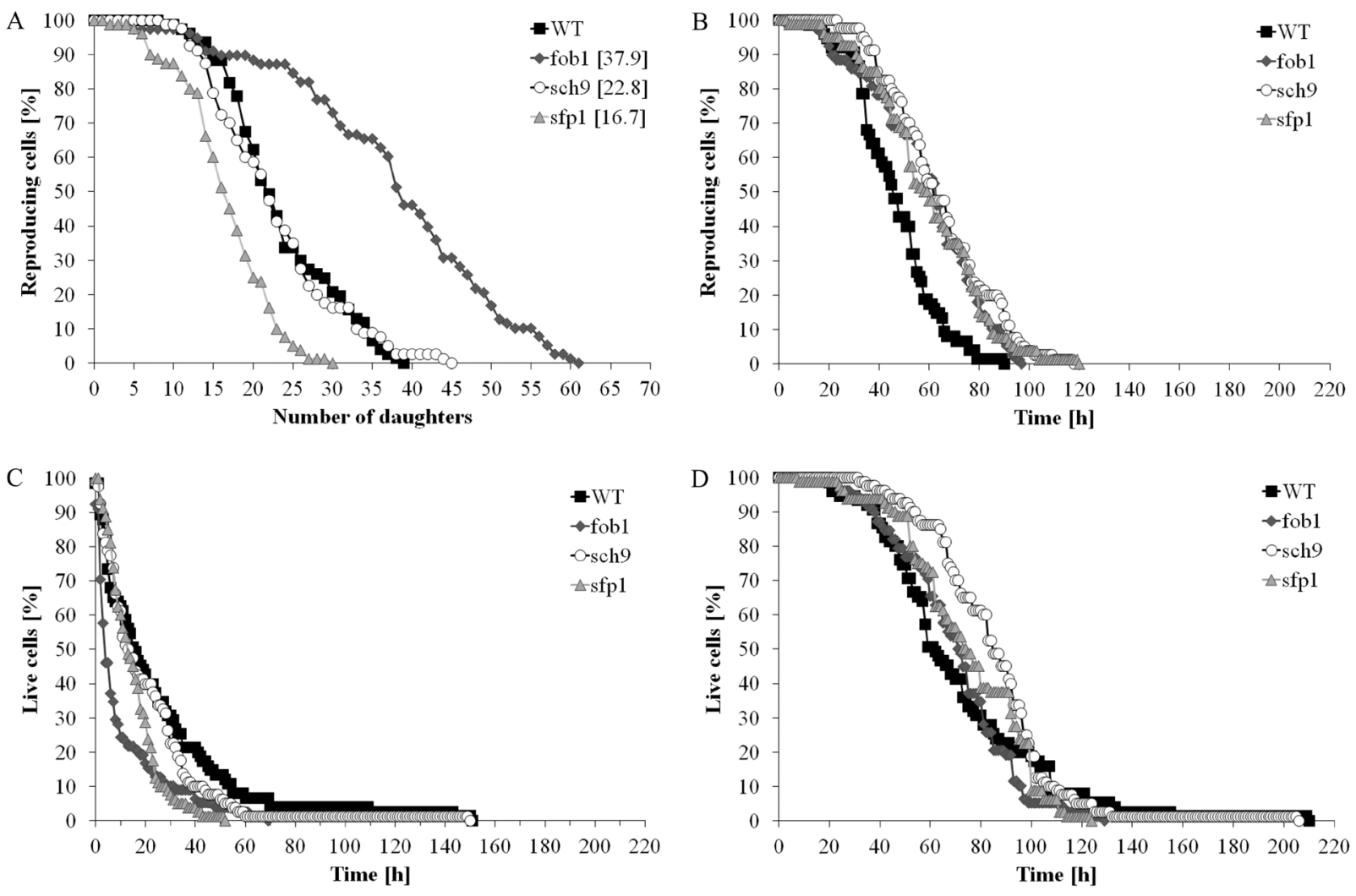

Figure 1. Comparison of the reproductive potential (A), reproductive lifespan (B), post-reproductive lifespan (C) and total lifespan (D) of the haploid wild-type yeast strain BMA64-1A and isogenic mutant strains fob 1 Values shown in parentheses are the mean values of the reproductive potential.

expressed in units of time). During the manipulation, the plates were kept at $28^{\circ} \mathrm{C}$ for $16 \mathrm{~h}$ and at $4^{\circ} \mathrm{C}$ during the night. The data represent the mean values from three separate experiments.

Estimation of cell volume. Cell volume was estimated by means of analysis of microscopic images recorded every fifth cell budding during the routine procedure of determining the reproductive potential. Images were captured with the Nikon Eclipse E200 microscope equipped with the Olympus DP26 digital camera. Cell diameter was measured using the Olympus cellSens Standard software. Cell diameter (d) was measured four times in various planes for each cell and the mean value was used for calculations. Assuming that each cell has a regular shape similar to the sphere, the cell volume was calculated as $4 / 3 \pi(d / 2)^{3}$.

Determination of mean doubling time. Mean doubling time was calculated individually for each of the analysed cells during routine determination of reproductive potential. During the calculations, the time of the two first reproductive cycles was not taken into account (in the case of virgin cells, the first as well as the second doubling time is longer than in older yeast cell); the time of the first reproductive cycle performed at the start of each day's experiment was also rejected (during the night the plates was kept at a low temperature of $+4^{\circ} \mathrm{C}$ ). The data represent the mean values from three independent experiments (with forty cells used in each experiment).

Statistics. Statistical analysis was performed with the use of StatSoft, Inc. (2011) (STATISTICA, data analysis software system, version 10. www.statsoft.com) using the $t$-test for independent samples in respect of the variable test and Dunnett's post hoc test. The results were presented as mean \pm standard deviation. Statistically significant differences were taken at $P<0.01$.

\section{RESULTS AND DISCUSSION}

The phenotypic effects of mutations often show a clear dependence on the genetic background, thus suggesting the need for an analysis of strains representing different genetic backgrounds. It is especially important in the analysis of reproductive potential where genetic background has an impact on the number of daughter cells produced (Dmello et al., 1994; Kaeberlein et al., 2005; Molon et al., 2015). In this study, we used three yeast mutants - fob $1 \Delta, \operatorname{sch} 9 \Delta$ and $\operatorname{sfp} 1 \Delta-$ representing one standard genetic background, i.e. BMA64-1A, where the characteristic feature is the large size achieved by the cells in comparison with cells representing other genetic backgrounds. All the analysed genes belong to the "longevity" genes but represent different mechanisms of action. Furthermore, to assess the impact of the genetic background on the analysed lifespan parameters, we compared the obtained results with those presented in another paper (Molon et al., 2015), where we used these mutants in the BY4741 genetic background.

To check the phenotypic effects of the analysed mutations, we determined the reproductive potential and lifespan expressed in units of time. First, we checked reproductive potential of the selected yeast strains, expressed as the number of daughters produced by a mother cell. Our results showed different values of reproductive po- 
Table 2. Mean Reproductive potential (number of generations), reproductive lifespan, post-reproductive lifespan and total lifespan of the yeast strains studied (mean \pm S.D., combined data from duplicate experiments). ${ }^{* *} p<0.01$ compared to the wild-type strain

\begin{tabular}{|c|c|c|c|c|}
\hline Strain & $\begin{array}{l}\text { Reproductive potential } \\
\text { [number of generations] }\end{array}$ & $\begin{array}{l}\text { Reproductive lifespan } \\
\text { [h] }\end{array}$ & $\begin{array}{l}\text { Post-reproductive lifespan } \\
\text { [h] }\end{array}$ & $\begin{array}{l}\text { Total lifespan } \\
\text { [h] }\end{array}$ \\
\hline BMA64-1A & $23.6 \pm 7$ & $46.4 \pm 18.9$ & $24.1 \pm 25.6$ & $70.5 \pm 32.8$ \\
\hline fob1 & $37 \pm 9.12^{* *}$ & $58.7 \pm 22.4^{* *}$ & $10.5 \pm 14.9^{* *}$ & $69.2 \pm 23.0$ \\
\hline $\operatorname{sch} 9 \Delta$ & $22.81 \pm 7.9$ & $64.3 \pm 21.1^{* *}$ & $18.5 \pm 15.3$ & $82.9 \pm 21.4^{* *}$ \\
\hline$s p p 1 \Delta$ & $16.74 \pm 5.6^{* *}$ & $59.4 \pm 22.3^{* *}$ & $14.9 \pm 10.2^{* *}$ & $74.4 \pm 24.3$ \\
\hline
\end{tabular}

tential for each of the analysed yeast strains, even though all of them were treated as "longevous": as such, they should have produced more daughter cells compared to the wild-type strain (Fig. 1A). The reproductive potential of the fob1 $\Delta$ mutant is higher, while that of $s p p 1 \Delta$ is statistically significantly lower in comparison with the wild-type strain. In turn, the SCH9 gene deletion has no effect on the reproductive potential in that genetic background (Table 2). The increased reproductive potential of the fob1s mutant was initially associated with low levels of ERCs (Defossez et al., 1999). However, the results obtained by Takehiko Kobayashi showed that despite the significant reduction in the amount of ERCs in one of the strains (rARS $\Delta-3$ ), the reproduction potential dropped. This was postulated to be the consequence of the rDNA instability; such instability occurs more frequently in mother cells rather than daughters (Ganley et al., 2009; Lindstrom et al., 2011). These results confirm a hypothesis which assumes that stability of the rDNA regulates the stability of the whole genome, and thus is an important factor in regulating the "longevity" of yeast (Kobayashi, 2008). The decrease of the reproductive potential in the case of $s f p 1 \Delta$ is consistent with the data obtained by Heeren and coworkers (Heeren et al., 2009) but differs significantly from the data obtained by Molon and coworkers (Molon et al., 2015) in the studies where the BY4741 background was used. Given that all of the analysed genes are defined as "longevity genes", these results tend to be somewhat surprising and suggest that the phenotypic effect of the mutations is strongly dependent on the genetic background.

For an explanation of the differences in the reproductive potential observed in the analysed BMA64-1A genetic background, we have studied changes in cell size during the entire reproductive period. That experimental

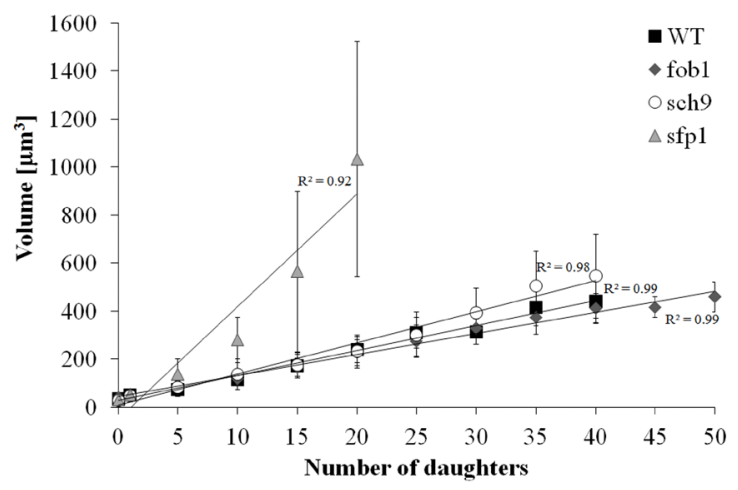

Figure 2. Dependence of cell volume on the number of daughters accomplished by mother yeast cells. The bars indicate S.D. approach was associated with the hypertrophy hypothesis, which explains the relationship between the reproductive potential and the cell volume growth rate per generation (Bilinski, 2012, Bilinski et al., 2012). The hypertrophy hypothesis assumes that limited reproductive potential is related to cell achieving its maximum volume (hypertrophy). Analysis of the kinetics of cell volume changes during successive cycles in the case of the wildtype yeast strain and selected mutants indicates an important role of cell volume in limiting the reproductive potential. The maximum volume of the fob1 $\Delta$ mutant cell remains at the same level as in the wild-type strain. However, its doubling time is shorter, hence the increase in size during single generation is lower, and therefore cells of that mutant produce more daughters. In the case of the $\operatorname{sch} 9 \Delta$ mutant, cells were larger compared to the wild-type and the fob1 $1 \Delta$ mutant cells. On the other hand, in the case of $s / p 1 \Delta$ the observed volume of the cells was the biggest (Fig. 2). Cells of that strain attain a huge volume, which consequently leads to their breakdown and destruction (not shown). A significant increase in the volume of $\operatorname{sch} 9 \Delta$ and $s p p 1 \Delta$ mutant cells may be associated with statistically significant prolongation of the doubling time (Fig. 3). This, in turn, may be explained by the fact that knock-out of the SFP1 and SCH9 genes slows down the growth rate of the yeast cells (Blumberg \& Silver, 1991, Toda et al., 1988). Both Sch9p and Sfp1p participate in regulation of ribosome biogenesis and RiBi genes transcription, which are essential for the cell (Jorgensen et al., 2004). Among the factors involved in ribosome biogenesis, the protein Sfp1 is the strongest START repressor because it regulates expression of numerous genes affecting the cell volume (Jorgensen et al., 2002). Furthermore, we suspect that hypertrophy of $s p p 1 \Delta$ and partially of $s c h 9 \Delta$ may be a result

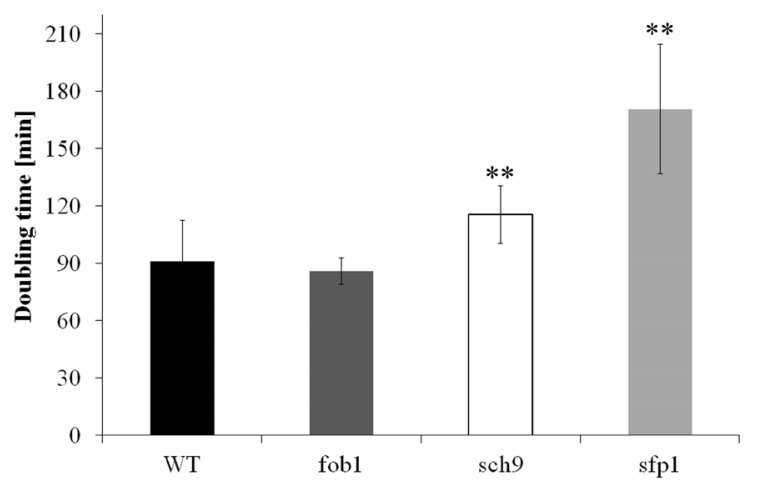

Figure 3. Comparison of average doubling time during reproduction of the haploid wild-type yeast strain BMA64-1A and isogenic mutant strains fob $1 \Delta$, sch $9 \Delta$ and sfp $1 \Delta$.

${ }^{* *} p<0.01$ compared to the wild-type strain. 
of certain problems with coordination of two processes, namely cell volume growth and budding. In this genetic background (BMA64-1A), a problem with the correct cell cycle process, caused e.g. by the mutation, will lead to an increased volume - a phenomenon that was not observed in other genetic backgrounds. The cracking of oversized cells explains significant reduction in the reproduction potential of the $s f p 1 \Delta$ mutant compared to the previous data obtained for the other genetic background, i.e. BY4741 (Molon et al., 2015).

As showed by the literature data and the results obtained in this study, reproductive potential is regulated by the rate of cell volume increase during successive reproducing cycles (Yang et al., 2011; Zadrag-Tecza et al., 2009). Therefore, the numerical dimension of the reproductive potential does not reflect the actual length of cell life. Yeast lifespan encompasses not only the time when the yeast cell reproduces (reproductive lifespan). After the end of the last cycle, the cell does not die but continues to live for a certain period of time, the length of which depends on the doubling time and the number of daughters produced by the mother cell (Zadrag et al., 2008). Disregarding that stage of yeast cell life may lead to some problems with interpretation of the results, especially when these results are strain-dependent and vary between genetic backgrounds. An analysis of the duration of different stages of yeast cell life, i.e. reproductive and post-reproductive phases, may provide useful information for identification of the role of genes in relation to reproductive potential and total lifespan.

The reproductive lifespan was statistically significantly extended in the case of all the analysed strains (Fig. 1b). Cells of the fob1 $\Delta$ mutant demonstrated a significant increase in their reproductive potentials; therefore, their reproductive lifespans also increased in comparison with the wild-type strain. The $s f p 1 \Delta$ mutant had a significantly decreased reproductive potential, while its reproductive lifespan was much longer compared to the wild-type strain. This extension of the reproductive phases of the sch9 and sfp1 mutants, with lack of differences in reproductive potential $(s c h 9)$, or even lack of reduction in reproductive potential $(s f p 1)$ (Fig. 1A), may be a consequence of the extension of the doubling time, clearly seen in the case of the spp1 mutant (Fig. 3). We then examined the lifetime after reproduction (post-reproductive lifespan). A significant shortening of that phase in the case of fob1s and $s f p 1 \Delta$ compared to the wildtype strain is associated with two different factors. The fob1 $\Delta$ mutant demonstrates an increase in reproductive potential; therefore, its post-reproductive lifespan is inversely proportional to the value of reproductive potential, as discussed previously (Zadrag-Tecza et al., 2013). In turn, $s p p 1 \Delta$ has a decreased reproductive potential and a very short post-reproductive lifespan, which might be explained by a significant increase in the cell volume during successive cycles and disintegration of cells. We did not observe changes of that parameter in the $\operatorname{sch} 9 \Delta$ mutant (Fig. 1C). The sum of the reproductive and postreproductive lifespans indicates the total lifespan of cells. In our study, only the $\operatorname{sch} 9 \Delta$ strain showed a statistically significant increase in the total lifespan. This was associated with a significant extension of the reproductive lifespan of that mutant, and only a slightly shortened post-reproductive phase. Interestingly, the fob1 $\Delta$ mutant performed 37 generations on average and lived as long as the sfp1 $1 \Delta$ strain which performed only 16 generations on average (Fig. 1D; Table 2).

The obtained data confirm our earlier observations that the phenotypic effect of the mutation is in many cases strain-dependent. In such a situation, it is difficult to draw any general conclusions without analysing the effect of a particular mutation in different genetic backgrounds. Even in the case of conserved pathways, such as Sch9p, the effect of the mutation may depend on the phenotypic properties of a wild-type strain. Further analysis should answer the question why we have seen such large variations in reproductive potential with the same mutants in different genetic backgrounds. It seems that some mutations may lead to dysregulation of the coordination between cell growth and budding. This may lead to an increase in the doubling time and to faster achievement of the state of hypertrophy. The results confirm earlier observations that reproductive potential (fertility) is strongly associated with cell volume growth per generation (Zadrag-Tecza et al., 2009; Bilinski et al., 2012). High hypertrophy can effectively reduce reproductive potential in yeast. Expressing lifespan of yeast not only in the number of daughters but also in units of time could provide a better explanation for the obtained results.

It seems that further studies are needed to strengthen this statement; however, the data presented in this work as well as in the previous one (Molon et al., 2015; Zadrag-Tecza et al., 2013) support it strongly.

\section{Acknowledgements}

We are grateful to Prof. Tomasz Bilinski for helpful discussions and critical review of the manuscript.

This work was supported by Grant no. DEC2013/09/B/NZ3/01352 from the Polish National Science Centre.

\section{SREFERENCES}

Aguilaniu H, Gustafsson L, Rigoulet M, Nystrom T (2003) Asymmetric inheritance of oxidatively damaged proteins during cytokinesis. Science 299: 1751-1753, http://dx.doi.org/10.1126/science.1080418.

Bilinski T (2012) Hypertrophy, replicative ageing and the ageing process. FEMS Yeast Res 12: 739-740, http://dx.doi.org/10.1111/ j.1567-1364.2012.00843.x.

Bilinski T, Zadrag-Tecza R, Bartosz G (2012) Hypertrophy hypothesis as an alternative explanation of the phenomenon of replicative aging of yeast. FEMS Yeast Res 12: 97-101, http://dx.doi. org/10.1111/j.1567-1364.2011.00759.x.

Bitterman KJ, Medvedik O, Sinclair DA (2003) Longevity regulation in Saccharomyces cerevisiae: linking metabolism, genome stability, and heterochromatin. Microbiology and molecular biology reviews: Microbiol Mol Biol Rev 67: 376-399, http://dx.doi.org/10.1128\%2FMM BR.67.3.376-399.2003.

Blumberg H, Silver P (1991) A split zinc-finger protein is required for normal yeast growth. Gene 107: 101-110.

Defossez PA, Prusty R, Kaeberlein M, Lin SJ, Ferrigno P, Silver PA, Keil RL, Guarente L (1999) Elimination of replication block protein Fob1 extends the life span of yeast mother cells. Mol Cell 3: $447-455$.

Dmello NP, Childress AM, Franklin DS, Kale SP, Pinswasdi C, Jazwinski SM (1994) Cloning and characterization of LAG1, a longevity-assurance gene in yeast. J Biol Chemi 269: 15451-15459.

Erjavec N, Larsson L, Grantham J, Nystrom T (2007) Accelerated aging and failure to segregate damaged proteins in Sir2 mutants can be suppressed by overproducing the protein aggregation-remodeling factor Hsp104p. Genes \& Develop 21: 2410-2421. http:/ /dx.doi. org/10.1101/gad.439307.

Fabrizio P, Pozza F, Pletcher SD, Gendron CM, Longo VD (2001) Regulation of longevity and stress resistance by Sch9 in yeast. Science 292: 288-290. http:/ /dx.doi.org/10.1126/science.1059497.

Ganley ARD, Ide S, Saka K, Kobayashi T (2009) The effect of replication initiation on gene amplification in the rDNA and its relationship to aging. Mol Cell 35: 683-693. http:/ /dx.doi.org/10.1016/j. molcel.2009.07.012

Gershon H, Gershon D (2000) The budding yeast, Saccharomyces cerevisiae, as a model for aging research: a critical review. Mech Ageing Develop 120: 1-22. http:/ /dx.doi.org/10.1016/s0047-6374(00)00182-2.

Heeren G, Rinnerthaler M, Laun P, Von Seyerl P, Koessler S, Klinger H, Hager M, Bogengruber E, Jarolim S, Simon-Nobbe B, Schueller 
C, Carmona-Gutierrez D, Breitenbach-Koller L, Mueck C, JansenDuerr P, Criollo A, Kroemer G, Madeo F, Breitenbach M (2009) The mitochondrial ribosomal protein of the large subunit, Afolp, determines cellular longevity through mitochondrial back-signaling via TOR1. Aging-Us 1: 622-636.

Jazwinski SM (1999) Longevity, genes, and aging: a view provided by a genetic model system. Exp Gerontol 34: 1-6.

Jorgensen P, Nishikawa JL, Breitkreutz BJ, Tyers M (2002) Systematic identification of pathways that couple cell growth and division in yeast. Science 297: 395-400. http:/ /dx.doi.org/10.1126/science. 1070850.

Jorgensen P, Rupes I, Sharom JR, Schneper L, Broach JR, Tyers M (2004) A dynamic transcriptional network communicates growth potential to ribosome synthesis and critical cell size. Genes $\&$ Develop 18: 2491-2505. http:/ /dx.doi.org/10.1101/gad.1228804.

Kaeberlein M (2010) Lessons on longevity from budding yeast. Nature 464: 513-519. http:/ /dx.doi.org/10.1038/nature08981.

Kaeberlein M, Kennedy BK (2005) Large-scale identification in yeast of conserved ageing genes. Mechanisms of ageing and development, 126: 17-21. http:/ /dx.doi.org/10.1016/j.mad.2004.09.013.

Kaeberlein M, Kirkland KT, Fields S, Kennedy BK (2005) Genes determining yeast replicative life span in a long-lived genetic background. Mecha Ageing Develop 126: 491-504. http:/ /dx.doi. $\operatorname{org} / 10.1016 /$ j.mad.2004.10.007.

Karathia H, Vilaprinyo E, Sorribas A, Alves R (2011) Saccharomyces cerevisiae as a model organism: a comparative study. PloS One 6: e16015. http:/ /dx.doi.org/10.1371/journal.pone.0016015.

Kobayashi T (2008) A new role of the rDNA and nucleolus in the nucleus-rDNA instability maintains genome integrity. Bioessays $\mathbf{3 0}$ 267-272. http:/ /dx.doi.org/10.1002/bies.20723.

Lindstrom DL, Leverich CK, Henderson KA, Gottschling DE (2011) Replicative age induces mitotic recombination in the ribosomal RNA gene cluster of Saccharomyces cerevisiae. PLoS Genetics 7: e1002015. http:/ /dx.doi.org/10.1371/journal.pgen.1002015.

Molon M, Zadrag-Tecza R, Bilinski T (2015) The longevity in the yeast Saccharomyces cerevisiae: A comparison of two approaches for assessment the lifespan. Biochemi Biophys Res Commun 460: 651-656. http:/ /dx.doi.org/10.1016/j.bbrc.2015.03.085.

Mortimer RK, Johnston JR (1959) Life span of individual yeast cells. Nature 183: 1751-1752. http:/ /dx.doi.org/10.1038/1831751a0.
Piper PW (2006) Long-lived yeast as a model for ageing research. Yeast 23: 215-226. http:/ /dx.doi.org/10.1002/yea.1354.

Reznick DN, Bryant MJ, Roff D, Ghalambor CK, Ghalambor DE (2004) Effect of extrinsic mortality on the evolution of senescence in guppies. Nature 431: 1095-1099. http:/ /dx.doi.org/10.1038/nature02936.

Sinclair D, Mills K, Guarente L (1998) Aging in Saccharomyces cerevisiae. Ann Rev Microbiol 52: 533-560. http:/ /dx.doi.org/10.1146/annurev. micro.52.1.533

Sinclair DA, Guarente L (1997) Extrachromosomal rDNA circles - a cause of aging in yeast. Cell 91: 1033-1042. http:/ /dx.doi. org/10.1016/s0092-8674(00)80493-6.

Steinkraus KA, Kaeberlein M, Kennedy BK (2008) Replicative aging in yeast: the means to the end. Ann Rev Cell Develop Biol 24: 29-54. http:/ /dx.doi.org/10.1146/annurev.cellbio.23.090506.123509.

Toda T, Cameron S, Sass P, Wigler M (1988) SCH9, a gene of Saccharomyces cerevisiae that encodes a protein distinct from, but functionally and structurally related to, cAMP-dependent protein kinase catalytic subunits. Genes \& Develop 2: 517-527.

Urban J, Soulard A, Huber A, Lippman S, Mukhopadhyay D, Deloche O, Wanke V, Anrather D, Ammerer G, Riezman H, Broach JR, De Virgilio C, Hall MN, Loewith R (2007) Sch9 is a major target of TORC1 in Saccharomyces cerevisiae. Mol Cell 26: 663-674. http:/ / dx.doi.org/10.1016/j.molcel.2007.04.020.

Yang J, Dungrawala H, Hua H, Manukvan A, Abraham L, Lane W, Mead H, Wright J, Schneider BL (2011) Cell size and growth rate are major determinants of replicative lifespan. Cell Cycle 10: 144-155. http:/ /dx.doi.org/10.4161/cc.10.1.14455.

Zadrag-Tecza R, Kwolek-Mirek M, Bartosz G, Bilinski T (2009) Cell volume as a factor limiting the replicative lifespan of the yeast Saccbaromyces cerevisiae. Biogerontology 10: 481-488. http:/ /dx.doi. org/10.1007/s10522-008-9192-0.

Zadrag-Tecza R, Molon M, Mamczur J, Bilinski T (2013) Dependence of the yeast Saccharomyces cerevisiae post-reproductive lifespan on the reproductive potential. Acta Biochim Pol 60: 111-115.

Zadrag R, Bartosz G, Bilinski T (2008) Is the yeast a relevant model for aging of multicellular organisms? An insight from the total lifespan of Saccharomyces cerevisiae. Curr Aging Sci 1: 159-165. 